\title{
Hole distribution in phononic crystals: Design and optimization
}

\author{
V. Romero-García and J. V. Sánchez-Pérez ${ }^{a)}$ \\ Centro de Technologías Físicas: Acústica, Universidad Politécnica de Valencia, Camino de Vera s/n, 46022 \\ Valencia, Spain \\ L. M. García-Raffi \\ Instituto Universitario de Matemática Pura y Aplicada, Universidad Politécnica de Valencia, Camino de \\ Vera s/n, 46022 Valencia, Spain

\begin{abstract}
J. M. Herrero, S. García-Nieto, and X. Blasco
Department of Systems Engineering and Control, Universidad Politécnica de Valencia, Camino de Vera $s / n$, 46022 Valencia, Spain
\end{abstract}

(Received 10 July 2008; revised 10 April 2009; accepted 10 April 2009)

\begin{abstract}
An exhaustive study has been made into the potential improvement in attenuation and focusing of phononic crystal arrays resulting from the deliberate creation of vacancies. Use is made of a stochastic search algorithm based on evolutionary algorithms called the epsilon variable multi-objective genetic algorithm which, in conjunction with the application of multiple scattering theory, enables the design of devices for effectively controlling sound waves. Several parameters are analyzed, including the symmetries used in the distribution of holes and the optimum number of holes. The validity and utility of the general rules obtained have been confirmed experimentally. (C) 2009 Acoustical Society of America. [DOI: 10.1121/1.3126948]
\end{abstract}

PACS number(s): 43.55.Ev, 43.20.El, 43.20.Hq, 43.40.Sk [KA]

Pages: $3774-3783$

\section{INTRODUCTION}

During the past decade, new materials for controlling sound waves have been successfully designed and used. Devices formed by arrays of scatterers embedded in another medium with a periodical modulation of the physical properties between them have been shown to be useful alternatives to homogeneous materials. The behavior of phononic crystals (PCs) formed from periodic arrays of cylinders in the range of wavelengths larger than the separation between the scatterers is a topic of increasing interest. In the ranges of frequencies related to the periodicity of the array formed, these systems present spectral band gaps where the propagation of sound is forbidden. A considerable effort has been made studying their physical properties, ${ }^{1-4}$ and this research has lead to the development of acoustic devices based on these materials. ${ }^{5-8}$

In recent years, there has been a growing interest in increasing and optimizing the sonic properties of these materials. The main motivation has been the development of technology for controlling sound. Some studies show performance improvements by means of varying the physical properties of the scatterers used: scatterers with resonant acoustic properties ${ }^{9-12}$ or acoustic absorption. ${ }^{13}$ Other possibilities include using scatterers with different shapes, ${ }^{14,15}$ or varying the position of the scatterers in order to change the properties of the array. In this context, some authors analyzed the use of high-symmetry quasicrystals as acoustic lenses. ${ }^{16}$

The properties of defect modes and their application as waveguides and acoustic filters have attracted a great deal of

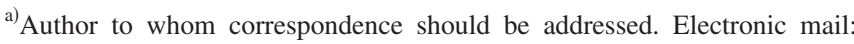
jusanc@ fis.upv.es
}

interest for many years, ${ }^{17-24}$ but the discussion of this mechanism and its properties are outside the scope of this work. Nevertheless, one of the strategies often used in the enhancement of the properties of PCs is based on the creation of defects in the starting array. Some authors demonstrated the possibility of increasing the attenuation capability of these materials by creating a periodic distribution of defects in the array. ${ }^{25}$ Other authors used the creation of vacancies in conjunction with optimization algorithms, such as the genetic algorithm, as a method to increase the attenuation properties or to create lenses based on PCs. ${ }^{26-29}$ There are clear advantages in using this method in the design of devices based on PCs, and this seems a suitable method for designing devices that are lighter and cheaper than complete PCs. Moreover, in some cases, as in the case of acoustic barriers, these new devices could be more competitive-both acoustically and economically-than the classical screens currently used. However, a study to analyze and systematize the best strategy for the mechanism that creates the holes seems necessary.

In this paper, we investigate the optimization of the acoustic properties of these materials using the mechanism for the creation of vacancies (subsequently referred to as "holes") in starting and complete two-dimensional (2D) PCs. Our aim is to provide general rules regarding (i) the optimum number of vacancies and (ii) how these vacancies should be distributed inside the crystal. Our approach to this problem consists of optimizing several acoustic properties simultaneously by means of a search algorithm. This situation is usually known as a multi-objective problem and may be solved with an optimization algorithm called the multiobjective evolutionary algorithm. ${ }^{30}$ In this work, we have used a new implementation of this tool based on genetic 
algorithms, termed the epsilon variable multi-objective genetic algorithm (evMOGA), ${ }^{31,32}$ in conjunction with a multiple scattering theory (MST). ${ }^{33}$ A parallel implementation of the evMOGA method is used here, and the execution time of the optimization process is drastically reduced. This approach has not been previously used in 2D PC design due to the fact that, until now, the optimization of the sonic properties of these materials has been considered as a single objective problem. ${ }^{26-29}$ The rest of the paper is arranged as follows. In Sec. II, we define the method of calculation. The results and discussion are developed in Sec. III. The experimental results that support our conclusions are presented in Sec. IV. Finally, we explain our conclusions in Sec. V.

\section{METHOD OF CALCULATION}

\section{A. MST}

Several mathematical methods have been used in recent years to study the physical behavior of PCs. These methods can be classified as theoretical or phenomenological. The former are based either on mathematical functions with a fixed symmetry or on a numerical resolution of the wave equation. $^{34,35}$ Phenomenological methods are based on experimental data obtained in specific situations. ${ }^{36}$ In this paper, we have used a theoretical method called MST.

When an incident sound wave travels inside a PC, a multiple scattering process is produced due to the periodicity of the scatterers in the array. MST (Refs. 34-37) is based on the well-known Korringa-Kohn-Rostoker theory ${ }^{38,39}$ and is a self-consistent theoretical method for calculating acoustic pressure. It includes all orders of scattering for mixed and high contrast composites. In this work, we have used a $2 \mathrm{D}$ MST for rigid cylinders with Neumann boundary conditions. The total acoustic pressure incident around the $n$th cylinder is the combined effect of the total acoustic incident pressure and the scattered pressure. Then,

$$
P_{\mathrm{in}}^{n}(\boldsymbol{r})=P_{0}(\boldsymbol{r})+\sum_{j=1, j \neq 1}^{N} P_{s}\left(\boldsymbol{r}, \boldsymbol{r}_{j}\right) .
$$

We can express the total acoustic pressure incident around the $j$ th cylinder as

$$
P_{\text {in }}^{n}(\boldsymbol{r})=\sum_{q=-\infty}^{q=\infty} B_{q}^{n} J_{q}\left(k\left|\boldsymbol{r}-\boldsymbol{r}_{n}\right|\right) e^{i q \theta_{\boldsymbol{r}-\boldsymbol{r}_{n}}}
$$

The incident pressure from the source with respect to the coordinates centered at $\boldsymbol{r}_{i}$ is

$$
P_{0}(\boldsymbol{r})=\sum_{q=-\infty}^{q=\infty} S_{q}^{n} J_{q}\left(k\left|\boldsymbol{r}-\boldsymbol{r}_{n}\right|\right) e^{i q \theta_{\boldsymbol{r}-\boldsymbol{r}_{n}},}
$$

and the scattered pressure is

$$
\begin{aligned}
P_{S}\left(\boldsymbol{r}, \boldsymbol{r}_{n}\right)= & \sum_{q=-\infty}^{q=\infty} \sum_{l=-\infty}^{l=\infty} i \pi A_{l}^{j} H_{l-q}\left(k\left|\boldsymbol{r}_{n}-\boldsymbol{r}_{j}\right|\right) e^{i(l-q) \theta_{\boldsymbol{r}_{n}-\boldsymbol{r}_{j}} J_{q}(k \mid \boldsymbol{r}} \\
& \left.-\boldsymbol{r}_{n} \mid\right) e^{i q \theta_{\boldsymbol{r}-\boldsymbol{r}_{n} .}}
\end{aligned}
$$

Using Eqs. (2)-(4) in Eq. (1), we obtain the following coefficient equation:

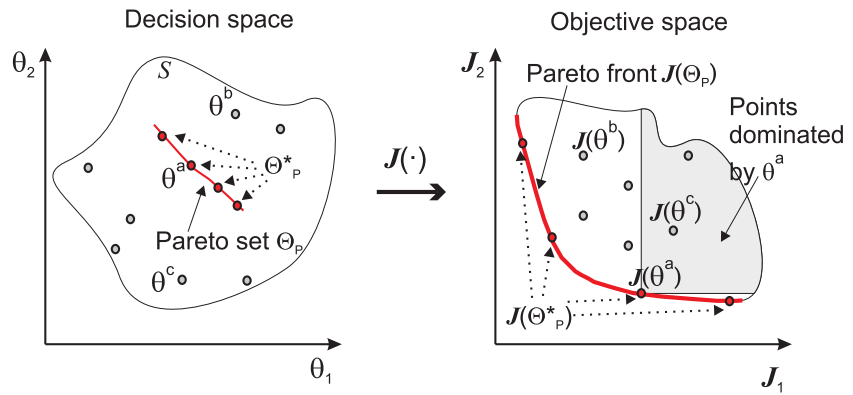

FIG. 1. (Color online) Example of a multiobjective optimization problem with $2 \mathrm{D}$ decision and objective space.

$$
B_{q}^{n}=S_{q}^{n}+\sum_{j=1, j \neq 1}^{N} \sum_{l=-\infty}^{l=\infty} i \pi A_{l}^{j} H_{l-q}\left(k\left|\boldsymbol{r}_{n}-\boldsymbol{r}_{j}\right|\right) e^{i(l-q) \theta_{\boldsymbol{r}_{n}-\boldsymbol{r}_{j}} .}
$$

To solve Eq. (5), we have to relate the coefficients $B_{q}^{j}$ and $A_{q}^{j}$. This can be achieved with the Neumann boundary condition:

$$
\left.\frac{\partial P_{\mathrm{ext}}}{\partial n}\right|_{r=r_{0}}=0
$$

where $n$ represents the normal direction of the boundary surface. The coefficients $A_{q}^{j}$ can then be obtained, and the acoustic pressure at any point outside the cylinders is

$$
P(\boldsymbol{r})=i \pi H_{0}^{(1)}(k|\boldsymbol{r}|)+\sum_{l=1}^{N} \sum_{q=-\infty}^{q=\infty} i \pi A_{q}^{l} H_{q}\left(k\left|\boldsymbol{r}-\boldsymbol{r}_{l}\right|\right) e^{i q \theta_{\boldsymbol{r}-\boldsymbol{r}_{l}},}
$$

where $N$ is the number of cylinders with radius $r$ located at $\boldsymbol{r}_{l}$ (with $1=1, \ldots, N$ ), $k$ is the wave number, $i$ is the imaginary unit, $H_{0}^{(1)}$ the zeroth order first-type Hankel function, $\theta_{\mathbf{r}-\boldsymbol{r}_{l}}$ the azimuthal angle of the vector $\boldsymbol{r}-\boldsymbol{r}_{l}$ to the positive $x$-axis, $A_{l q}$ are the coefficients of the series expansion of the pressure, and $H_{l q}$ is the $q$ th order first-type Hankel function.

Although MST can be used to calculate accurately the dispersion relation of mixed composites where the contrast between the material parameters of scatterers and the host is very high ${ }^{40}$ the MST method presents some problems from the point of view of its use in classical optimization methods due to the large set of parameters involved: the type of dependencies, the size of the decision space (number of possible configurations of structures, i.e., PCs with or without holes), and the computational time show the complexity of the problem. The coefficients of the series expansions in the MST, $A_{q}^{l}$, are determined numerically from the equations obtained using the boundary conditions, and their values are related to both the frequency and the parameters defining the PC. As a consequence, the acoustic pressure, Eq. (7), depends simultaneously on discrete and continuous variables and is difficult to optimize.

An optimization method looks for the best solution in the decision space (also known as search space, see Fig. 1), that is, the space occupied by all the possible configurations of structures in our problem. Each structure is characterized by a vector of dimension $N$, where each coordinate is related with a position in the starting $\mathrm{PC}$, so that the value of each 
coordinate represents the existence of a cylinder, or a hole, in each position of the starting PC. Then, the dimension of the decision space is $N$ and the size is $2^{N}$. We also have to take into account that the computational time to calculate $A_{q}^{l}$ increases to the third power of the number of cylinders (dimension of the decision space), $N^{3}$. So, the large existing number of cylinders in the designed devices implies considerable computational time. Finally, difficulty is added by the fact that the pressure calculated by MST depends on several variables of different types (real, integer, etc.). Therefore, the use of classical optimization methods is not feasible. Fortunately, the parallel implementation of the evMOGA method used in this work enables us to overcome these problems.

\section{B. Multi-objective evolutionary algorithm}

Decisions about optimal design in many scientific or engineering areas involve searching for compromises between different objectives. It is natural to look for the best solution to each objective. However, if objectives are in conflict, an improvement in one, or more, objectives means a worsening in one, or more, of the other objectives. The difficulty is the absence of a single optimal solution. Problems where several conflicting objectives have to be optimized simultaneously are known in the literature as multi-objective optimization problems. For this type of problem, the optimal solution is a set called the Pareto set $\Theta_{p}$. The main characteristic of the members of this set is that any of the solutions is better than the other solutions for some of the objectives-meaning that all solutions are optimal in some sense. Basic concepts for these kinds of problems are illustrated in Fig. 1 for a minimization problem with two objectives $\left(J_{1}\right.$ and $\left.J_{2}\right)$ and two decision variables $\left(\theta_{1}\right.$ and $\left.\theta_{2}\right)$. The problem is set as

$$
\min J(\theta)=\min \left[J_{1}(\theta), J_{2}(\theta)\right] \text { subject to } \theta=\left[\theta_{1}, \theta_{2}\right] \in S .
$$

One of the important definitions in this tool is the concept of dominance: a point $\theta^{x}=\left[\theta_{1}^{x}, \theta_{2}^{x}\right]$ is dominated by another point $\theta^{y}=\left[\theta_{1}^{y}, \theta_{2}^{y}\right]$ if at least one of the objectives of $\theta^{x}$ $\left[J_{1}\left(\theta^{x}\right)\right.$ or $\left.J_{2}\left(\theta^{x}\right)\right]$ is worse than the corresponding $\theta^{y}$ objective $\left[J_{1}\left(\theta^{y}\right)\right.$ or $\left.J_{2}\left(\theta^{y}\right)\right]$. This is true providing the rest of the objectives are equal.

Figure 1 shows, as an example, a situation where $\theta^{a}$ dominates $\theta^{c}$, but not $\theta^{b}$. The gray area represents all the points (in the objective space) dominated by $\theta^{a}$.

We can define the Pareto set (and its corresponding Pareto front) as the set of nondominated points. The Pareto set in Fig. 1 is referred to as $\Theta_{p}$ and the Pareto front as $J\left(\Theta_{p}\right)$.

Solving a multi-objective optimization problem by obtaining the Pareto optimal solution (Pareto set and Pareto front) is not a trivial problem. For instance, some problems present an infinite number of points, and these problems must be solved by means of classical multi-objective optimization algorithms. ${ }^{41}$ In essence, the aim of these algorithms is to obtain a discrete approximation of the Pareto set $\Theta_{p}^{*}$ and Pareto front $J\left(\Theta_{p}^{*}\right)$ in a distribution near $J\left(\Theta_{p}\right)$, while ensuring that solutions are not too near each other (since they would then be more or less the same) and attempting to characterize all of the Pareto front (see Fig. 1). However, classical algorithms are sometimes not the best option in problems where the shape of the Pareto front is complicated and shows problems of discontinuity.

An interesting alternative for solving multi-objective optimization problems is based on the use of evolutionary algorithms, which enable the simultaneous generation of several elements of the Pareto optimal set in parallel and in a single run. Evolutionary algorithms (or evolutionary computations) are inspired by biological evolution: reproduction, mutation, recombination, and selection. ${ }^{30}$ An initial population (a set of possible solutions) evolves by applying genetic operators that combine the characteristics of some of the individuals of the population. At each iteration of the algorithm, the population changes and tries to converge to the optimal solution $\Theta_{p}, J\left(\Theta_{p}\right)$.

A number of authors have developed different operators or strategies for converting the original single objective evolutionary algorithms into multi-objective optimization evolutionary algorithms that converge toward the Pareto optimal set with a set that is sufficiently discrete and diverse to be able to characterize it. ${ }^{42}$ The good results obtained with this type of algorithm, together with their ability to handle a wide variety of problems with differing degrees of complexity, explains their increasing use in many situations. ${ }^{43}$

The algorithm used in this work is one of the most recent developments in MOGA. The evMOGA is an elitist multi-objective evolutionary algorithm based on the concept of epsilon-dominance. ${ }^{44}$ A complete and detailed version of the evMOGA algorithm is described in Refs. 31 and 32. The evMOGA algorithm obtains a discrete approximation of the Pareto set, $\Theta_{p}^{*}$, that converges toward the Pareto optimal set $\Theta_{p}$ in a smart way (in this type of distribution the more sloped a zone of the Pareto front, then the more points that are used to characterize the zone), and using limited memory resources.

One of the limitations of multi-objective optimization evolutionary algorithms is their high computational cost. For each individual of the chosen population, the objective functions have to be computed, and in most cases, this represents the costly part of the algorithm. Improvements in execution time have been obtained with a parallel implementation of evMOGA. Several alternatives for parallelization are possible, ${ }^{45}$ and we have chosen the master-slave configuration. In this architecture, one processor works as master, executing the evolutionary tasks of evMOGA, while the other processors evaluate the objective function (see Fig. 2).

The master has to send a point in the decision space (or a set of points) to each slave, which then perform an objective function evaluation, and returns the results to the master. The master works synchronously, waiting for all the objective function values from each slave. After receiving all the values, the master performs the evolution to produce the next iteration and then sends the new population to the slaves for evaluation. This type of implementation is the simplest and does not change evMOGA operators or behavior. Thus, the optimization process is divided into three main procedures: evolution, communication between master-slaves, and the objective function evaluation. If the evolution and the 


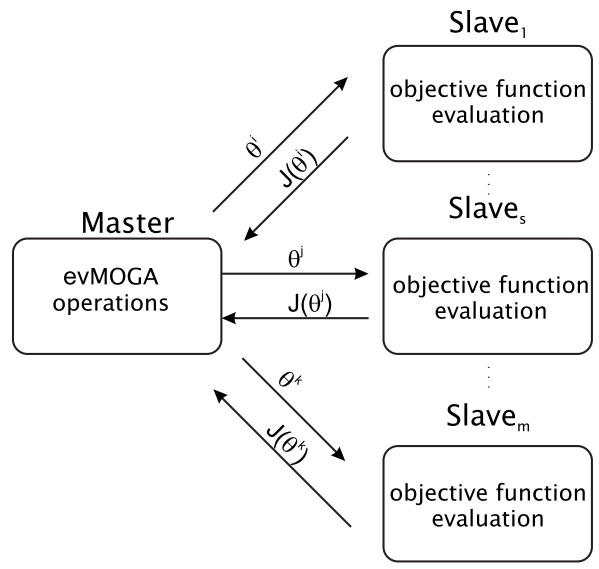

FIG. 2. Master-slave architecture for parallel evMOGA.

master-slave communication do not have a high computational cost, the overall time is theoretically divided by the number of slaves. So, the master-slave configuration offers a significant saving of time.

\section{Starting conditions: Strategies in the creation of holes}

In this paper, we seek to improve the acoustic properties of 2D PCs formed by isolated and rigid cylinders in air. We chose a starting PC containing 73 (4 cm diameter) cylinders, positioned in seven rows in a triangular pattern with a lattice constant $a=6.35 \mathrm{~cm}$. We have calculated, by means of MST, the acoustic pressure of the optimized samples for a predetermined range of frequencies, at a point located $1 \mathrm{~m}$ from the middle of the PC and in the $\Gamma X$ direction (Fig. 3).

The optimized structures are obtained by means of the creation of vacancies, i.e., removing cylinders in the starting PC. To design these structures, we have used four strategies in the creation of vacancies by taking into account the symmetry axis of the starting PC (Fig. 3): (i) symmetry around the $X$ axes $(\operatorname{sym} X)$, (ii) symmetry around the $Y$ axes $(\operatorname{sym} Y)$, (iii) symmetry around both the $X$ and $Y$ axes $(\operatorname{sym} X Y)$, and (iv) random (nosym).

\section{Characterization of the devices obtained}

Due to the nature of multi-objective problems, the optimized solution is represented by a set of structures (Pareto set, see Sec. II B). It seems necessary to define suitable tools to characterize these optimized structures. Moreover, these
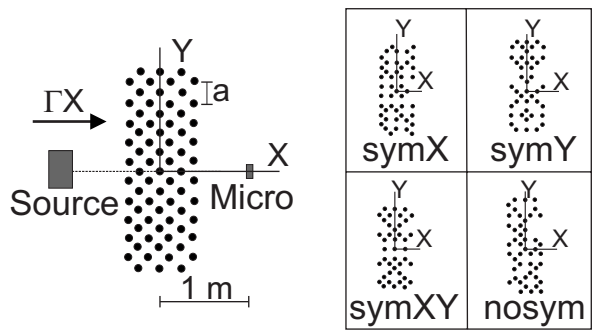

FIG. 3. Starting conditions for the analysis. The inset shows an example of each of the different strategies used in the creation of vacancies in the starting PC. tools will enable a comparison among the different optimized samples. We have defined both optimizing and structural tools. The former help arrange the resulting samples as a function of their acoustic properties. These tools are Pareto front $(\mathrm{PF})$, optimizing factor $\left(O_{f}\right)$, and area of the spectrum (AS). Structural tools help establish the best arrangement of the vacancies for enhancing the acoustic properties of the resulting devices. These parameters are fraction of vacancies $\left(F_{v}\right)$ and asymmetry $(A)$. These tools are defined as follows.

Pareto front (PF). As we have mentioned above, in the approach to multi-objective optimization problems, the notion of optimized structures changes because the solution is a good compromise between the objectives involved in an optimization process. Thus, we do not normally obtain a single solution, but a set of solutions called the Pareto optimal set, which is mapped by the objective functions of the Pareto front (see Fig. 1). All points representing the PF correspond to optimum solutions. However, in our case, with two objective functions, we can see in Fig. 1 that an optimal solution with respect to one of the objective functions implies a low optimization with respect to the other function. Therefore, we need to define some decision criteria to choose the most suitable solution. This solution is characterized by the definition of the $\boldsymbol{Q}$-vector that is a point of the PF. If we consider $k$ objective functions, the $\boldsymbol{Q}$-vector is defined in such a way that its distance to the origin is minimal in the objective space,

$$
\boldsymbol{Q} / \min \left\{\sqrt{\sum_{i=1}^{k} J_{i}(\boldsymbol{Q})^{2}}\right\} .
$$

This point is a compromise between all the objective functions optimized, and offers the best stability with respect to the optimization procedure.

Optimizing factor $\left(O_{f}\right)$. This parameter helps determine how much better each optimization is with respect to the other optimizations, quantifying the improvement of the optimization process. Given several Pareto fronts for the same optimization problem, we define the optimization factor $\left(O_{f}\right)$ as the Euclidean distance between the points $\boldsymbol{Q}$ of any two different PFs. Thus, if we consider $\boldsymbol{Q}_{1}$ and $\boldsymbol{Q}_{2}$ as two PF $Q$-vectors, then

$$
O_{f}=\sqrt{\sum_{i=1}^{N}\left(Q_{1 i}-Q_{2 i}\right)^{2}} .
$$

An important parameter used to measure the improvement obtained by the Pareto fronts is the so-called ideal point. ${ }^{46}$ The ideal point is defined as the vector formed by the lowest components among all points in the PF. That is, the first components of the ideal point are the minimum value of all first components of the points in the PF; the second component is obtained in the same way. Distances to the ideal point, measured in the objective space, are one of the classical solution quality indices in multi-objective optimization. However, the ideal point is not easy to find in practice. The $Q$-vector that corresponds to a real structure is easy to find in practice, and it is near to the value of the ideal point in our optimization problem. In addition, $\boldsymbol{Q}$-vector provides infor- 
mation about the goodness of the optimization results.

Area of spectrum (AS). This is an important parameter based on the acoustic spectrum generated by the considered sample. It is defined as the area enclosed between the positive range of spectra, and the $0 \mathrm{~dB}$ threshold line in the range of selected frequencies. This parameter measures the sonic capability of the considered structure, and increasing its value means an improvement in sonic properties. This parameter has been used in previous works for attenuation cases. $^{29}$

Fraction of vacancies $\left(F_{v}\right)$. This structural parameter provides information about the number of existing vacancies in the resulting sample. We define this parameter as the number of vacancies with respect to the total number of cylinders:

$$
F_{v}=\frac{N_{v}}{N}
$$

where $N_{v}$ is the number of created vacancies, and $N$ is the total number of cylinders in the starting PC. $F_{v}$ belongs to the interval $[0,1]$.

Asymmetry (A). This structural parameter enables the distribution of the created vacancies in the resulting samples to be determined-taking into account the asymmetry of the existence of each vacancy with respect to the symmetry axes of the starting PC $(X$ and $Y)$. We define this parameter as

$$
A=\frac{A_{x}+A_{y}}{2},
$$

where $A_{i}(i=x, y)$ represents the asymmetry with respect to the $i$-axis, which is defined as

$$
A=\frac{\sum_{j=1} N \gamma_{A}\left(b_{j}-b_{j}^{\prime}\right)}{2 N_{v}},
$$

$b_{j}$ and $b_{j}$ take the values 1 or 0 -depending on the existence, or not, of the cylinder in position $j$, or in the symmetrical $j$, with respect to a symmetry axis of the starting PC. $N_{v}$ is again the number of created vacancies. The parameters $b$ and $b^{\prime}$ are binary vectors with dimensions equal to the total number of cylinders $N$. Thus, we have defined the asymmetry distribution function $\gamma_{A}$ as

$$
\gamma_{A}(x)= \begin{cases}1 & \text { if } x \neq 0 \\ 0 & \text { if } x=0\end{cases}
$$

With these definitions, $A$ belongs to the interval $[0,1]$.

General rules for building optimized devices for each sonic application based on these tools is given in Sec. III.

\section{RESULTS AND DISCUSSION}

In this section, we discuss the improvement of two main PC sonic properties by means of the creation of vacancies. Specifically, we want to independently increase their attenuation and their focusing properties. In each case, we are interested in optimizing, several spectrum properties. Because of this, we have considered these situations as multiobjective optimization problems to be resolved using the evMOGA method. The methodology followed involves the generation of vacancies in a starting PC using four different symmetry strategies, as explained above, so that the optimized samples present fewer cylinders than the starting PC.

evMOGA works by minimizing the functions under study (objective functions). Two objective functions based on the pressure values obtained by the MST have been used in both attenuation and focusing cases. The resulting samples that present the best values of the two objective functions are selected by evMOGA when forming the Pareto front. The analysis of the PF, and the other tools defined above, will enable us to approach the best strategy.

Multiple execution of evMOGA has been performed to increase the reliability of the results. Four runs were made for each of the analyzed symmetries. The first execution starts with differing constraints and initial populations, but takes into account the starting PC. The following runs start with the best structures found in the previous runs.

\section{A. Attenuation sound devices}

The first step in our attenuation study consists of defining the objective functions chosen for optimizing the acoustic attenuation of the starting $\mathrm{PC}$, in a predetermined point of measurement, for a range of frequencies ( $f$ $=[2300,3700] \mathrm{Hz}$ analyzed at intervals of $\Delta f=50 \mathrm{~Hz}$ ) and in the $\Gamma X$ direction. We are interested in maximizing the acoustic attenuation level and stability. In other words, we want to obtain a high attenuation level in a predetermined range of frequencies, with the least possible fluctuation in the attenuation spectrum.

Acoustic attenuation at a point $(x, y)$, due to an incident plane wave of frequency $f$ traveling through a PC formed by an array of scatterers of radius $r_{l}$ placed at $\left(X_{\text {cyl }}, Y_{\text {cyl }}\right)$ coordinates, is defined as

$$
\text { Attenuation }(\mathrm{dB})=20 \log \left\{\frac{1}{\left|p\left(x, y, X_{\mathrm{cyl}}, Y_{\mathrm{cyl}}, f, r_{l}\right)\right|}\right\},
$$

where $p$ is the value of the acoustic pressure and is obtained by means of the MST. Attenuation is normalized for an incident acoustic pressure equal to unity. From Eq. (15), it is easy to conclude that maximizing sound attenuation means minimizing acoustic pressure. Taking into account this fact, we have defined two objective functions, $J_{1}$ and $J_{2}$, representing the mean pressure and the mean deviation, respectively, in the range of frequencies considered,

$$
\begin{aligned}
& J_{1}(\boldsymbol{x})=\bar{p}=\frac{\sum_{i=1}^{N_{f}}\left|p_{i}(\boldsymbol{x})\right|}{N_{f}}, \\
& J_{2}(\boldsymbol{x})=\sqrt{\frac{\sum_{i}\left(\left|p_{i}(\boldsymbol{x})-\bar{p}\right|\right)^{2}}{N_{f}^{2}}},
\end{aligned}
$$

where $N_{f}$ represents the number of frequencies considered in the range under study $\left(N_{f}=29\right.$, in our case $)$ and $\mathbf{x}$ $=\left(X_{\text {cyl }}, Y_{\text {cyl }}\right)$ represents the variable under study, meaning the position of the existing cylinders in the sample. Minimizing these functions implies obtaining a maximum for the acoustic attenuation in the chosen range of frequencies. $J_{1}$ is related with the attenuation level in Eq. (16), and $J_{2}$ represents 

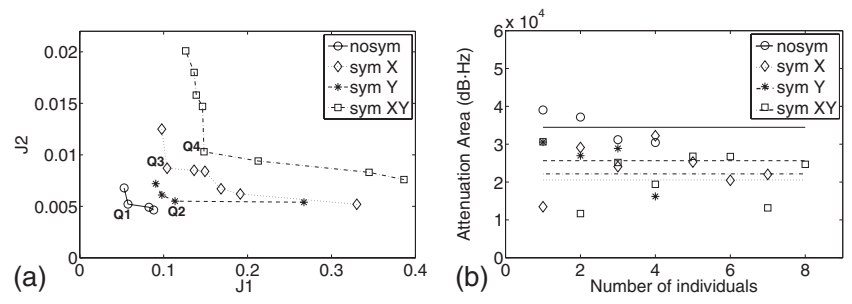

FIG. 4. (a) Pareto fronts for the different symmetries of generation of vacancies used for attenuation effect; (b) attenuation area for each analyzed symmetry.

the stability of the attenuation level in the range of considered frequencies in Eq. (17). We consider the attenuation level as the truly important function in this study-stability being a refinement of the obtained results, as shown below.

Figure 4(a) shows the Pareto fronts for the results obtained by evMOGA with the four vacancy generation strategies used. Their position in the plot implies the improved capability of the obtained attenuation properties. The closer the Pareto fronts are to the origin of the coordinates, the better the optimization. This is due to the fact that a low $J_{1}$ implies a high attenuation level through Eq. (16), and a low $J_{2}$ represents lower mean deviation. We can arrange the strategies used as a function of the results shown: nosym $>\operatorname{sym} Y>\operatorname{sym} X>\operatorname{sym} X Y$. It can be seen that the nosym strategy presents the highest optimization levels for the objective functions, and $\operatorname{sym} X Y$ seems the worst.

Moreover, we can compare the acoustic attenuation level for both the optimized samples and the starting PC. To do this, we will use the optimizing factor parameter. Taking into account that the $\boldsymbol{Q}$-vector for the PF corresponding to the nosym strategy is $\left(J_{1}, J_{2}\right)=(0.0575,0.0052)$, and the values for the starting PC are $J_{1}=0.4633$ and $J_{2}=0.056$, we can obtain the value of the optimizing factor, $O_{f}=0.4090$. It seems that the attenuation level $\left(J_{1}\right)$ is the truly important function in our study. Because for both cases, the difference between their values is similar to the $O_{f}$ value. So, it seems that $J_{2}$ plays a secondary role in our attenuation study, representing the stability of the attenuation level. Finally, the value of $O_{f}$ indicates that, physically, the creation of vacancies in a starting PC seems a suitable strategy for improving the attenuation characteristics of PCs. Thus, the value of $O_{f}$ means that, in acoustic terms, there is a difference in the attenuation capacity between the starting $\mathrm{PC}$ and the best sample of the nosym strategy value at $18.5 \mathrm{~dB}$.

After looking at Fig. 4(a), it seems possible to quantify the differences between the acoustic attenuations obtained among the four considered strategies. Regarding the $Q$-vectors of the best (nosym) and the worst $(\operatorname{sym} X Y)$ strategies, the value of the $O_{f}$ parameter is $O_{f}=0.0907$. This value represents an attenuation difference of $8.2 \mathrm{~dB}$ between both optimized samples.

To quantify how much better each strategy of vacancy generation is when compared to the others, we have analyzed the area of spectrum (AS) parameter, called in this case, attenuation area (AA). This parameter has been calculated for the spectra of all the optimized samples shown in Fig. 4(a). The points in Fig. 4(b) represent the value of the AA
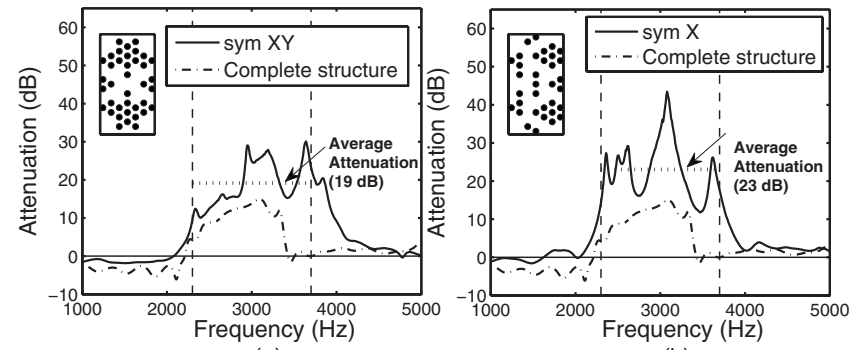

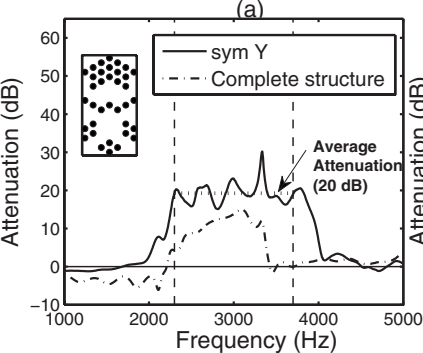

(c)

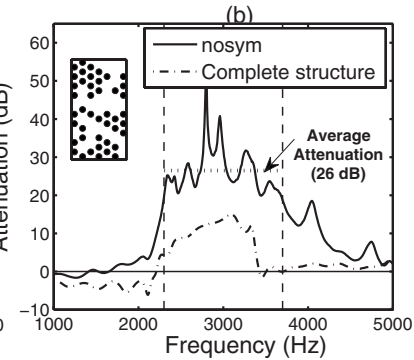

(d)
FIG. 5. Spectra of the resulting optimized samples: (a) Q1, (b) Q2, (c) Q3, and (d) Q4 shown in Fig. 4(a) (continuous line), and of the starting PC (dotted line). The range of frequencies optimized is delimited by two vertical dashed lines. The attenuation average level in the optimized range of frequencies for each of the symmetries used is represented (horizontal dotted line). Configurations of the optimized samples corresponding to each Q-vector are included as an inset.

parameter for each resulting optimized structure in each of the symmetries of vacancies used, and the lines represent each average value of the parameter. The biggest AA corresponds to the nosym strategy, and this fact means that the resulting optimized samples obtained with this strategy show the best improvement in their attenuation properties.

Figure 5 can help us understand Figs. 4(a) and 4(b). Here, we present the attenuation spectra of the resulting samples marked in Fig. 4 (a) as $Q_{1}, Q_{2}, Q_{3}$, and $Q_{4}$, corresponding to the $\boldsymbol{Q}$-vectors of each strategy of vacancy generation-analyzed together with that corresponding to the starting PC. The best average, as we have mentioned above, corresponds to the nosym strategy. Note that the values of the objective functions for the resulting samples shown in Fig. 4(a) are in very good agreement with the MST spectra simulations shown in Fig. 5. Thus, we can compare the improvement of both $J_{1}$ (mean pressure) in Fig. 4(a) and the average attenuation in Fig. 5. In Fig. 4(a), it can be seen that $J_{1}^{Q_{4}}>J_{1}^{Q_{2}}>J_{1}^{Q_{3}}>J_{1}^{Q_{1}}$, and this is in good agreement with the results obtained in Fig. 5: nosym $>\operatorname{sym} Y>\operatorname{sym} X>\operatorname{sym} X Y$. It can also be observed that the improvement of $J_{2}$ in the process is not as significant as the improvement of $J_{1}$. In Fig. 5 the predominance of the attenuation level criterion in the attenuation spectra of the resulting samples can be seen.

In short, taking into account the results obtained for the optimizing tools defined in Sec. II D (PF, SA, and $O_{f}$ ), the nosym strategy for creating vacancies in a starting PC seems the most suitable method for improving the acoustic attenuation properties of PCs. A possible explanation may be that evMOGA produces an optimized combination of holes; therefore, the multiple scattering process is able to increase the acoustic attenuation. To attenuate a range of frequencies, a large combination of distances between scatterers inside the PC is necessary to obtain an acoustic attenuation greater 

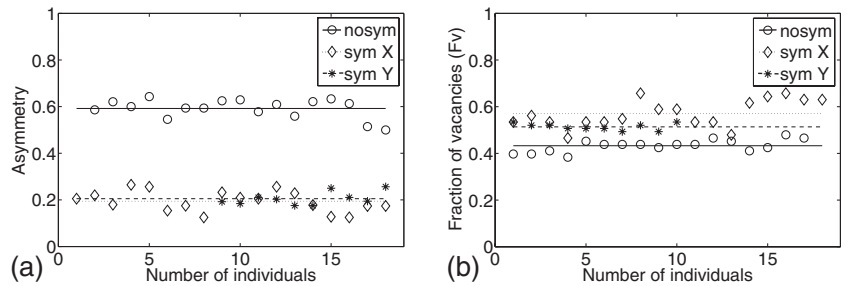

FIG. 6. (a) Asymmetry parameter and (b) fraction of vacancies for each analyzed symmetry. The points represent the value of the parameter for each of the optimized structures obtained, and the line represents their average value.

than that corresponding to the starting PC modulating the phase. Among the four analyzed symmetries, the random symmetry presents more varying distances and, consequently, more attenuated frequencies.

The next step consists in obtaining some general rules about the number of vacancies and their distribution on the starting PC, as required to obtain structures with optimized attenuation properties. This has been achieved using the structural tools defined in Sec. II D: the fraction of vacancies $\left(F_{v}\right)$ and asymmetry $(A)$. In Fig. 6(a), we show the values of the asymmetry parameter for each of the analyzed symmetries in the creation of vacancies. In the case of the nosym strategy, it can be seen that the optimized value of the asymmetry is around $60 \%$. This result indicates the method to create vacancies in the starting PCs to optimize attenuation properties, according to the definition of asymmetry. Note that the corresponding value of this parameter for the sym $X Y$ vacancies is $A_{\text {symXY }}=0$ due to the definition of asymmetry. Moreover, taking into account the Pareto fronts shown in Fig. 4(a), sym $X Y$ becomes the worst strategy to increase the attenuation properties of PCs; therefore, the value of the $A$ parameter is in good agreement with the results obtained with the PF. Accordingly, only the three remaining symmetries have been analyzed.

Figure 6(b) shows the results of the study of the fraction of vacancies. This parameter gives us the optimum number of vacancies for each of the considered strategies. It can be seen that for the best strategy (nosym) obtained above, the value of this parameter is the highest, and it is around $43 \%$ of the total number of cylinders in the starting PC. We have shown in this section, by means of the use of both optimization and structural parameters, that (i) the creation of vacancies is a suitable method for increasing the acoustic attenuation properties of PCs and (ii) the nosym strategy seems the best method for creating vacancies. Moreover, we have obtained general rules for creating random (nosym) vacancies in a PC based on the structural parameters defined. The optimal values of $A$ and $F_{v}$ to build an optimal structure should be $59 \%$ of asymmetry and $43 \%$ of vacancies. These values correspond to the best devices in terms of improved acoustic attenuation.

\section{B. Focusing sound devices}

This section examines the optimization of the focusing properties of PCs by means of the creation of vacancies in a starting PC. Specifically, the aim is to increase the fo-
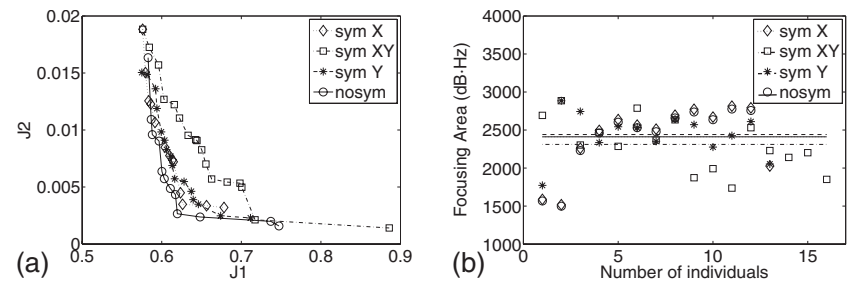

FIG. 7. (a) Pareto fronts for the different vacancy generation symmetries used for focusing effect; (b) focusing area for each analyzed symmetry. The points represent the value of the parameter for each of the optimized structures obtained and the line represents their average value.

cusing properties of these materials, in a predetermined point of measurement, for a range of frequencies $(f$ $=[1400,2000] \mathrm{Hz}$, analyzed at intervals of $\Delta f=50 \mathrm{~Hz})$ and in the $\Gamma X$ direction. Note that the chosen range of frequencies is just below the first band gap, that is, inside the first transmission band. As in the case of attenuation optimization, we have defined two objective functions, taking into account that the aim is to maximize the acoustic pressure at the predetermined point. The acoustic focusing level is defined as

$$
\text { Focalization }(\mathrm{dB})=20 \log \left\{\left|p\left(x, y, X_{\mathrm{cyl}}, Y_{\mathrm{cyl}}, f, r_{l}\right)\right|\right\},
$$

where $p$ again means acoustic pressure calculated by MST. As in the case of acoustic attenuation, the objective is to obtain a high pressure level with a small fluctuation of the pressure spectrum. Thus, the objective functions $J_{3}$ and $J_{2}$ to minimize are defined as

$$
\begin{aligned}
& J_{3}(\boldsymbol{x})=\frac{1}{\bar{p}}=\frac{N_{f}}{\sum_{i=1}^{N_{f}}\left|p_{i}(\boldsymbol{x})\right|}, \\
& J_{2}(\boldsymbol{x})=\sqrt{\frac{\sum_{i}\left(\left|p_{i}(\boldsymbol{x})-\bar{p}\right|\right)^{2}}{N_{f}^{2}}},
\end{aligned}
$$

where $\boldsymbol{x}=\left(X_{\mathrm{cy}}, Y_{\mathrm{cy}}\right)$ and $N_{f}$ are defined as in the attenuation case. Note that $J_{2}$ is the same objective function used in the optimization case explained above, and this means the stability of the pressure level is in the range of the considered frequencies. The minimization of $J_{3}$ implies a high level pressure (focusing effect). Again, the pressure level has been considered as the main parameter in this study, pushing stability into the background of the obtained results.

Figure 7(a) shows the Pareto fronts with the optimization results using the four strategies for creating vacancies obtained by evMOGA for the focusing case. Here, as in the case of the attenuation analysis, the same order can be seen in the strategies used as a function of the obtained optimization: nosym $>\operatorname{sym} Y>\operatorname{sym} X>\operatorname{sym} X Y$. Figure 7(a) shows that the nosym strategy offers higher optimization levels for the objective functions, and sym $X Y$ offers the worst strategy. However, due to the small separation between all the Pareto fronts, it can be concluded that the vacancy creation strategy chosen to optimize the focusing properties of the PCs is unimportant. This result is in agreement with the reasoning and the strategy followed by several authors ${ }^{25}$ in the case of focusing optimization. Moreover, the creation of vacancies 


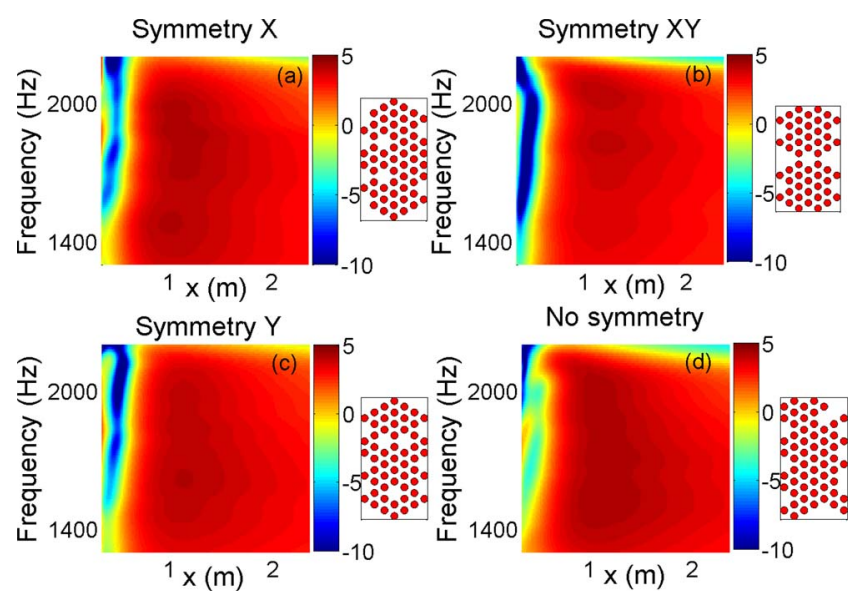

FIG. 8. (Color online) Acoustic level in the acoustic focus zone. On the $O X$ axis, the distance along the direction of the incident wave is shown, and frequencies for the four strategies analyzed on the $O Y$ axis are also shown. The color bar represents the acoustic level in decibels. The corresponding samples are also included.

seems less efficient in the optimization of the focusing than in the case of attenuation. Again, we can evaluate the optimization process by comparing the values of the $O_{f}$ parameter between the nosym strategy and the starting PC. The $Q$-vector for the nosym strategy is $\left(J_{3}, J_{2}\right)$ $=(0.6196,0.0027)$ and the corresponding objective function for the starting PC is $J_{3}=0.6428, J_{2}=0.0437$. The calculated value of the optimizing factor is $O_{f}=0.0471$. This means an improvement in the focusing properties equal to $0.4280 \mathrm{~dB}$. In this case, $O_{f}$ means a low optimization level compared with that obtained in the attenuation case $\left(O_{f}=0.4090\right.$ meaning $18.5 \mathrm{~dB}$ ) in the focusing properties of the PC.

To support these results, we can analyze the area of spectrum (AS) tool, refered to as the focusing area (FA). This optimization parameter has been calculated in the same way as the attenuation case. Figure 7(b) shows the FA for the analyzed strategies, and the similarity between the average values for all the strategies used can be seen.

Figure 8 enables the above results to be collaborated. This figure shows increases in the acoustic level in the range of frequencies under study, and for the predetermined point of measurement of the coordinates $(1,0)$. It can be clearly seen that the difference in acoustic level between the analyzed symmetries is smaller when compared with the acoustic attenuation case.

We have studied the behavior of the structural tools in order to provide some general rules about the number of vacancies and their distribution on the starting PC when intending to optimize focusing properties.

Figure 9(a) shows the variation of the asymmetry parameter for each of the analyzed strategies. As in the attenuation case, the value for the nosym symmetry is around 0.6, and for other symmetries it is between 0.38 and 0.45 . Figure 9(b) shows the results regarding the fraction of vacancies necessary to optimize the focusing properties of PCs. For all cases, this parameter is around $20 \%$ of the total number of cylinders in the starting PC.

Taking into account the results obtained using both optimization and structural parameters, it can be concluded that
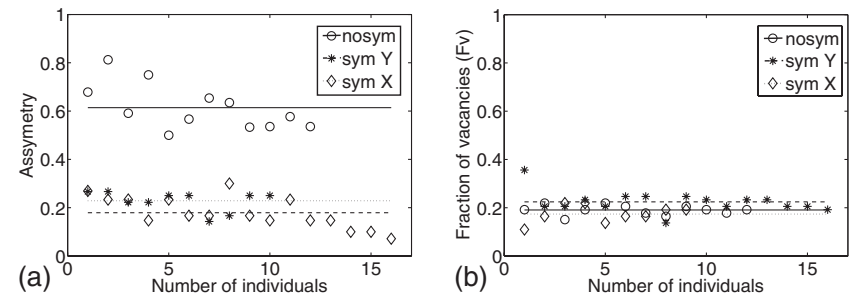

FIG. 9. (a) Asymmetry parameter and (b) fraction of vacancies for the analyzed symmetries. The points represent the value of the parameter for each of the optimized structures obtained, and the line represents their average value.

although the creation of vacancies provides an improvement in the focusing properties of PCs, this increase is smaller than that obtained in the attenuation case. Moreover, the strategy of vacancy creation does not seem a decisive factor in this optimization process.

\section{EXPERIMENTAL RESULTS}

We have shown that the sonic properties of PCs can be increased by creating vacancies. We have provided general rules for optimizing the attenuation and focusing behavior of these materials at a predetermined point and for $\Gamma X$ direction ( 0 of incidence). Both the mean pressure and the mean deviation of the sonic pressure are specifically used as objective functions. This section experimentally confirms the applicability and the robustness of the optimization rules obtained in Secs. II and III. To achieve this, we have designed the following random acoustic attenuation experiment. We have built a starting PC with an external shape and number of cylinders that differ from the PC used in the theoretical development. In this PC, we have created vacancies by removing a predetermined number of cylinders each time. We then measured the sound attenuation spectrum (the difference between the sound level recorded at the same point with and without the sample-usually called insertion loss) in the $\Gamma X$ direction (0). We also calculated the AA, $F_{v}$, and $A$ parameters for each of the resultant samples obtained. The experiments were performed in an anechoic chamber using a directional white noise sound source. Specifically, we used a PC with a honeycomb external shape, made from 397 cylinders of hollow aluminum with $4 \mathrm{~cm}$ diameters, $1 \mathrm{~m}$ long, and placed in a triangular array with parameter $a=6.35 \mathrm{~cm}$ (Fig. 10). Ten samples were created as we created vacancies by removing 40 cylinders each time.

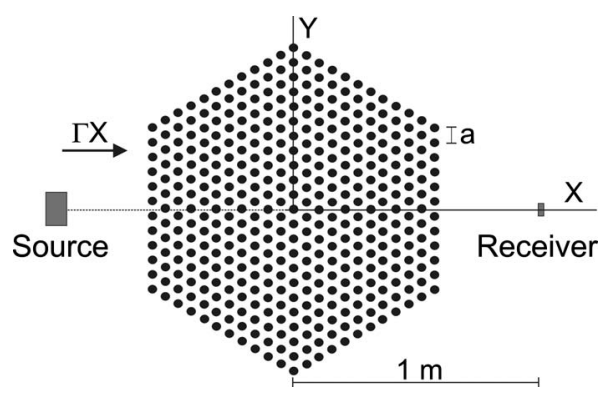

FIG. 10. Experimental measurement setup. The starting PC consists of hollow aluminum rods $1 \mathrm{~m}$ long and $4 \mathrm{~cm}$ in diameter arranged in a triangular pattern with constant lattice $a=6.35 \mathrm{~cm}$. There are 397 cylinders. 


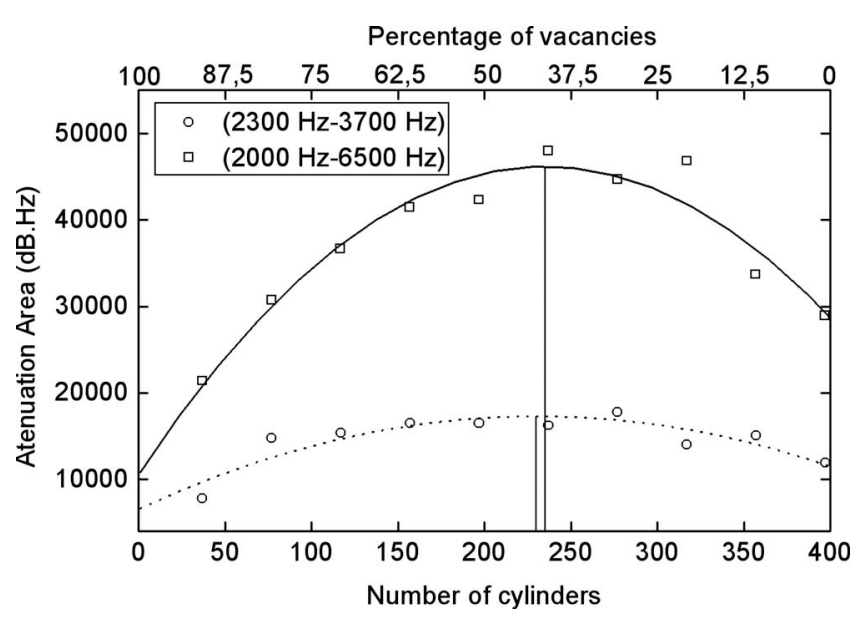

FIG. 11. Attenuation area versus number of cylinders for both analyzed cases.

In Fig. 11 we show the value of the AA parameter as a function of the number of cylinders, and for the two ranges of frequencies analyzed $(2300-3700 \mathrm{~Hz}$ and $2000-6500 \mathrm{~Hz}$ ). In both cases, it can be seen that there is a maximum of AA for a percentage of the existing vacancies of around $40 \%$ of the total cylinders of the starting PC. This value agrees with the value obtained in the optimization process.

The variation of the asymmetry parameter of the samples as a function of the number of vacancies is plotted in Fig. 12. It can be seen that the asymmetry is near $60 \%$ for the optimum number of vacancies corresponding to the best samples (around 40\%) obtained in both analyzed cases. Again, this value is in good agreement with the theoretical result obtained in the optimization study.

Attenuation spectra for the best optimized sample (larger AA) and the complete starting PC are plotted in Fig. 13 - corresponding to the range of frequencies from 2300 to $3700 \mathrm{~Hz}$ in Fig. 13(a) and from 2000 to $6500 \mathrm{~Hz}$ in Fig. 13(b). The best sample is also shown in each inset. In both cases, the increase of the AA when compared to the starting PC can be seen in those samples with vacancies.

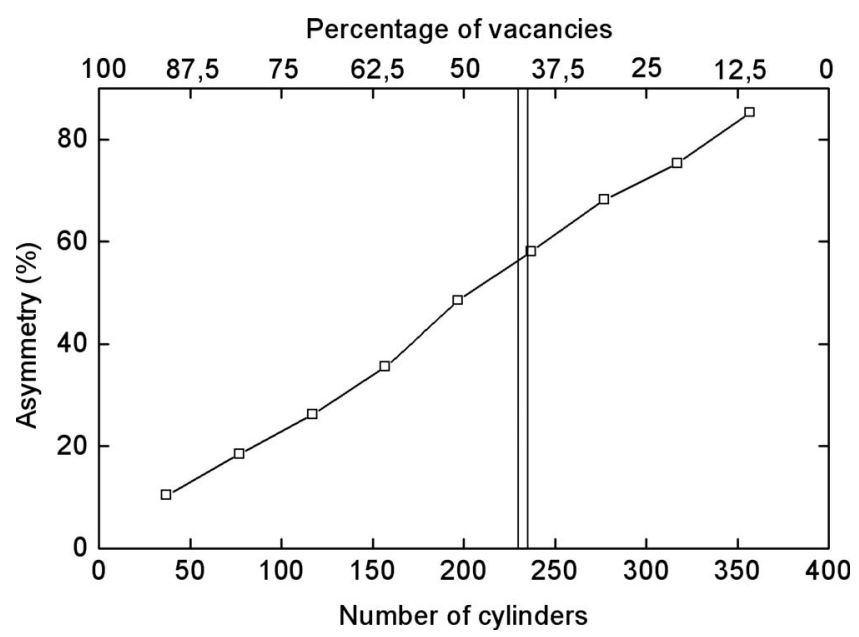

FIG. 12. Variation of the asymmetry of the nine obtained structures as a function of the vacancies created. Vertical lines represent the optimum number of vacancies for both analyzed cases.
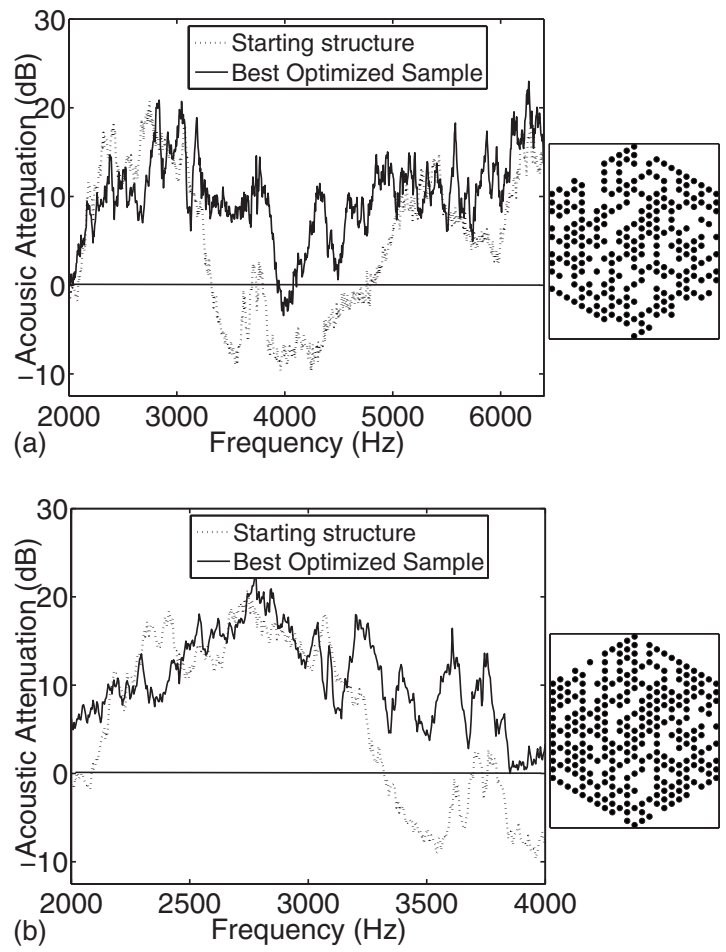

FIG. 13. Comparison of attenuation spectra corresponding to the starting PC, and the best sample obtained by means of the creation of vacancies: (a) $(2300-3700 \mathrm{~Hz})$; (b) $(2000-6500 \mathrm{~Hz})$. The corresponding best structure obtained is shown in the inset.

In short, the validity of the general rules obtained has been experimentally shown in our theoretical research on optimization. Moreover, the devices built by means of the creation of vacancies in the starting PC, when taking into account the theoretical general rules obtained, offer the best attenuation capability - in the predetermined range of frequencies analyzed theoretically; and also in a wider range.

\section{SUMMARY}

Based on the numerical results obtained by means of evMOGA in conjunction with MST, we have analyzed a mechanism for the creation of vacancies in a starting PC as a tool for increasing acoustic properties. The PC is formed by isolated cylinders surrounded by air. Specifically, we have studied the optimization of the attenuation and focusing properties of these materials and interesting conclusions have been obtained. First, these acoustic phenomena are not the same from the optimization point of view. Therefore, the creation of vacancies seems a suitable method for the improvement of attenuation capability, but not for improving focusing properties. Second, we have presented general rules for constructing attenuation devices based on PCs in an optimal approach: (i) the strategy used in the creation of vacancies seems an important factor and we have concluded that the random strategy is best; (ii) the optimal number of created vacancies is around $40 \%$ of the total number of cylinders in the starting PC, and the optimal asymmetry, i.e., the approach for distributing the vacancies, is near $60 \%$. Moreover, we have experimentally demonstrated the validity of these theoretical rules and we have shown that the obtained results are independent of the PC characteristics (external 
shape and number of cylinders) and the acoustic parameters to optimize (range of frequencies). Finally, due to the range of applicability of the wave crystal theory, the obtained results could be used as a guide for constructing devices in several wave fields.

\section{ACKNOWLEDGMENTS}

This work was partially supported by MEC (Spanish Government) and FEDER funds: Project Nos. 419DPI200507835, and MAT2006-03097 and Generalitat Valenciana Project Nos. GV06/026 and 420 GV/2007/191. The authors would like to thank John Rawlins (associate member of the Institute of Translation and Interpreting (No. 9743) of UK, and "Certified PRO" of ProZ according to the EN 15038) for his help with the use of English.

${ }^{1}$ J. Sánchez-Pérez, D. Caballero, R. Martínez-Sala, C. Rubio, J. SánchezDehesa, F. Meseguer, J. Llinares, and F. Glvez, "Sound attenuation by a two-dimensional array of rigid cylinders," Phys. Rev. Lett. 80, 5325-5328 (1998).

${ }^{2}$ W. M. Robertson and J. F. Rudy III, "Measurement of acoustic stop bands in two-dimensional periodic scattering arrays," J. Acoust. Soc. Am. 104, 694-699 (1998).

${ }^{3}$ M. Sigalas and E. Economou, "Attenuation of multiple-scattered sound," Europhys. Lett. 36, 241-246 (1996).

${ }^{4}$ F. Cervera, L. Sanchis, J. Sánchez-Prez, R. Martínez-Sala, C. Rubio, F. Meseguer, C. Lopez, D. Caballero, and J. Sánchez-Dehesa, "Refractive acoustic devices for airborne sound," Phys. Rev. Lett. 88, 023902 (2001). ${ }^{5} \mathrm{M}$. Kushwaha, "Stop-bands for periodic metallic rods: Sculptures that can filter the noise," Appl. Phys. Lett. 70, 3218-3220 (1997).

${ }^{6} J$. Sánchez-Pérez, C. Rubio, R. Martínez-Sala, R. Sánchez-Grandia, and V. Gómez, "Acoustic barriers based on periodic arrays of scatterers," Appl. Phys. Lett. 81, 5240-5242 (2002).

${ }^{7}$ L. S. Chen, C. H. Kuo, and Z. Ye, "Acoustic imaging and collimating by slabs of sonic crystals made from arrays of rigid cylinders in air," Appl. Phys. Lett. 85, 1072-1074 (2004).

${ }^{8}$ J. Bravo-Abad, T. Ochiai, and J. Sánchez-Dehesa, "Anomalous refractive properties of a two-dimensional photonic band-gap prism," Phys. Rev. B 67, 115116 (2003).

${ }^{9}$ Z. Liu, X. Zhang, Y. Mao, Y. Zhu, Z. Yang, C. Chan, and P. Sheng, "Locally resonant sonic materials," Science 289, 1734-1736 (2000).

${ }^{10}$ M. Hirsekorn, P. Delsanto, N. Batra, and P. Matic, "Modelling and simulation of acoustic wave propagation in locally resonant sonic materials," Ultrasonics 42, 231-235 (2004).

${ }^{11}$ M. Hirsekorn, "Small-size sonic crystal with strong attenuation bands in the audible frequency range," Appl. Phys. Lett. 84, 3364-33676 (2004).

${ }^{12} \mathrm{X}$. Hu and C. Chan, "Two-dimensional sonic crystals with Helmholtz resonators," Phys. Rev. E 71, 055601(R) (2005).

${ }^{13} \mathrm{O}$. Umnova, K. Attenborough, and C. Linton, "Effects of porous covering on sound attenuation by periodic arrays of cylinders," J. Acoust. Soc. Am. 119, 278-284 (2006).

${ }^{14}$ W. Kuang, Z. Hou, and Y. Liu, "The effects of shapes and symmetries of scatterers on the phononic band gap in 2D phononic crystals," Phys. Lett. A 332, 481-490 (2004).

${ }^{15}$ R. Min, Fugen Wu, L. Zhong, H. Zhong, S. Zhong, and Y. Liu, "Extreme acoustic band gaps obtained under high symmetry in 2D phononic crystals," J. Phys. D: Appl. Phys. 39, 2272-2276 (2006).

${ }^{16} \mathrm{X}$. Zhang, "Universal non-near-field focus of acoustic waves through highsymmetry quasicrystals," Phys. Rev. B 75, 024209 (2007).

${ }^{17}$ R. James, S. Woodley, C. Dyer, and V. Humphrey, "Sonic bands, bandgaps, and defect states in layered structures-theory and experiment," J. Acoust. Soc. Am. 97, 2041-2047 (1995).

${ }^{18} \mathrm{M}$. Sigalas, "Elastic wave band gaps and defect states in two-dimensional composites," J. Acoust. Soc. Am. 101, 1256-1261 (1997).

${ }^{19}$ M. Sigalas, "Defect states of acoustic waves in a two dimensional lattice of solid cylinders," J. Appl. Phys. 84, 3026-3030 (1998).

${ }^{20} \mathrm{~F}$. Wu, Z. Hou, Z. Liu, and Y. Liu, "Point defect states in two-dimensional phononic crystals," Phys. Lett. A 292, 198-202 (2001).
${ }^{21}$ J. Munday, C. Brad Bennett, and W. Robertson, "Band gaps and defect modes in periodically structured waveguides," J. Acoust. Soc. Am. 112, 1353-1358 (2002).

${ }^{22}$ A. Khelif, A. Choujaa, B. Djafari-Rouhani, M. Wilm, S. Ballandras, and $\mathrm{V}$. Laude, "Trapping and guiding of acoustic waves by defect modes in a full-band-gap ultrasonic crystal," Phys. Rev. B 68, 214301 (2003).

${ }^{23}$ A. Khelif, M. Wilm, V. Laude, S. Ballandras, and B. Djafari-Rouhani, "Guided elastic waves along a rod defect of a two-dimensional phononic crystal," Phys. Rev. E 69, 067601 (2004).

${ }^{24} \mathrm{~F}$. Wu, J. Liu, Y. Liu, and Y. Liu, "Splitting and tuning characteristics of the point defect modes in two-dimensional phononic crystals," Phys. Rev. E 69, 066609 (2004).

${ }^{25}$ D. Caballero, J. Sánchez-Dehesa, R. Martínez-Sala, C. Rubio, J. V. Sánchez-Pérez, L. Sanchis, and F. Meseguer, "Suzuki phase in twodimensional sonic crystals," Phys. Rev. B 64, 064303 (2001).

${ }^{26}$ A. Hakansson, J. Sánchez-Dehesa, and L. Sanchis, "Acoustic lens design by genetic algorithms," Phys. Rev. B 70, 214302 (2004).

${ }^{27}$ A. Hakansson, F. Cervera, and J. Sánchez-Dehesa, "Sound focusing by flat acoustic lenses without negative refraction," Appl. Phys. Lett. 86, 054102 (2005).

${ }^{28}$ A. Hakansson, J. Sánchez-Dehesa, and F. Cervera, "Experimental realization of sonic demultiplexing devices based on inverse designed scattering acoustic elements," Appl. Phys. Lett. 88, 163506 (2006).

${ }^{29}$ V. Romero-García, E. Fuster, L. M. García-Raffi, E. A. Sánchez-Pérez, M. Sopena, J. Llinares, and J. V. Sánchez-Pérez, "Band gap creation using quasiordered structures based on sonic crystals," Appl. Phys. Lett. 88, 174104 (2006).

${ }^{30}$ T. Back, Evolutionary Algorithms in Theory and Practice: Evolution Strategies, Evolutionary Programming, Genetic Algorithms (Oxford University Press, New York, 1996).

${ }^{31}$ J. Herrero, X. Blasco, M. Martínez, C. Ramos, and J. Sanchis, "Robust identification of a greenhouse model using multi-objective evolutionary algorithms," Biosyst. Eng. 98, 335-346 (2007).

${ }^{32}$ J. Herrero, M. Martínez, J. Sanchis, and X. Blasco, "Well-distributed pareto front by using the epsilon-moga evolutionary algorithm," Lect. Notes Comput. Sci. 4507, 292-299 (2007).

${ }^{33} \mathrm{~V}$. Twersky, "Multiple scattering of radiation by an arbitrary configuration of parallel cylinders," J. Acoust. Soc. Am. 24, 42-46 (1951).

${ }^{34} Y$. Chen and Z. Ye, "Theoretical analysis of acoustic stop bands in twodimensional periodic scattering arrays," Phys. Rev. E 64, 036616 (2001).

${ }^{35}$ D. García-Pablos, M. Sigalas, F. M. de Espinosa, M. Torres, M. Kafesaki, and N. García, "Theory and experiments on elastic band gaps," Phys. Rev. Lett. 84, 4349-4352 (2000).

${ }^{36}$ E. Fuster-Garcia, V. Romero-García, J. V. S. Pérez, L. M. García-Raffi, and E. A. S. Pérez, "A phenomenological model for sonic crystals based on artificial neural networks," J. Acoust. Soc. Am. 120, 636-641 (2006).

${ }^{37}$ M. Kafesaki and E. Economou, "Multiple scattering theory for threedimensional periodic acoustic composites," Phys. Rev. B 60, 11993 (1999).

${ }^{38}$ J. Korringa, "On the calculation of the energy of a Bloch wave in a metal," Physica (Amsterdam) 13, 392-400 (1947).

${ }^{39}$ W. Kohn and N. Rostoker, "Solution of the Schrodinger equation in periodic lattices with an application to metallic lithium," Phys. Rev. 94, 11111120 (1954).

${ }^{40}$ L. Sanchis, F. Cervera, J. Sánchez-Dehesa, J. V. Sánchez-Pérez, C. Rubio, and R. Martínez-Sala, "Reflectance properties of two-dimensional sonic band gap crystals," J. Acoust. Soc. Am. 109, 2598-2605 (2001).

${ }^{41} \mathrm{~K}$. Miettinen, Nonlinear Multiobjective Optimization (Kluwer Academic, Boston, 1998).

${ }^{42}$ C. Coello, D. Veldhuizen, and G. Lamont, Evolutionary Algorithms for Solving Multiobjective Problems (Kluwer Academic, Dordrecht, 2002).

${ }^{43}$ C. Coello and G. Lamont, Applications of Multiobjective Evolutionary Algorithms (Wold Scientific, Singapore, 2004).

${ }^{44}$ M. Laumanns, L. Thiele, K. Deb, and E. Zitzler, "Combining convergence and diversity in evolutionary multi-objective optimization," Evol. Comput. 10, 263-282 (2002).

${ }^{45} \mathrm{R}$. Ursem, "Models for evolutionary algorithms and their applications in systems identification and control optimization," Ph.D. thesis, University of Aarhus, Denmark (2003).

${ }^{46}$ M. Ehrgott and D. Tenfelde-Podehl, "Computation of ideal and Nadir values and implications for their use in MCDM methods," Eur. J. Oper. Res. 151, 119-139 (2003). 